\title{
Microwaves improve chromosome G-banding in fresh blood and bone marrow
}

F Solé, S Woessner

\begin{abstract}
A simple technique for obtaining good chromosomal G-banding on fresh blood and bone marrow is presented. The usual technique is modified by using a microwave oven before staining the preparations with Wright's stain.
\end{abstract}

$(f$ Clin Pathol 1992;45:1118)

Servei d'Hematologia

i Oncologia 1973,

Laboratori de

Citologia

Hematològica,

Hospital Central

L'Alianca, Av. San

Antoni Ma Claret 200,

08025 Barcelona,

Spain

F Solé

$\mathrm{S}$ Woessner

Correspondence to:

Dr Francesc Solé

Accepted for publication

16 April 1992

One of the technical problems in cytogenetics consists of obtaining good G-bands immediately after fresh slide preparations have been made. This does not present a problem in peripheral blood samples from healthy patients (constitutional karyotype), the chromosomes being adequate for bands of good quality to be obtained even on the same day as the slides have been prepared. But in haematological neoplasias the chromosomes obtained from peripheral blood and bone marrow preparations are usually of poor quality and fuzzy, with good G-bands difficult to obtain. If the G-bands are made immediately after preparing the slides, destruction or poor chromosome resolution are both common problems. To prevent this, some authors suggest a delay of

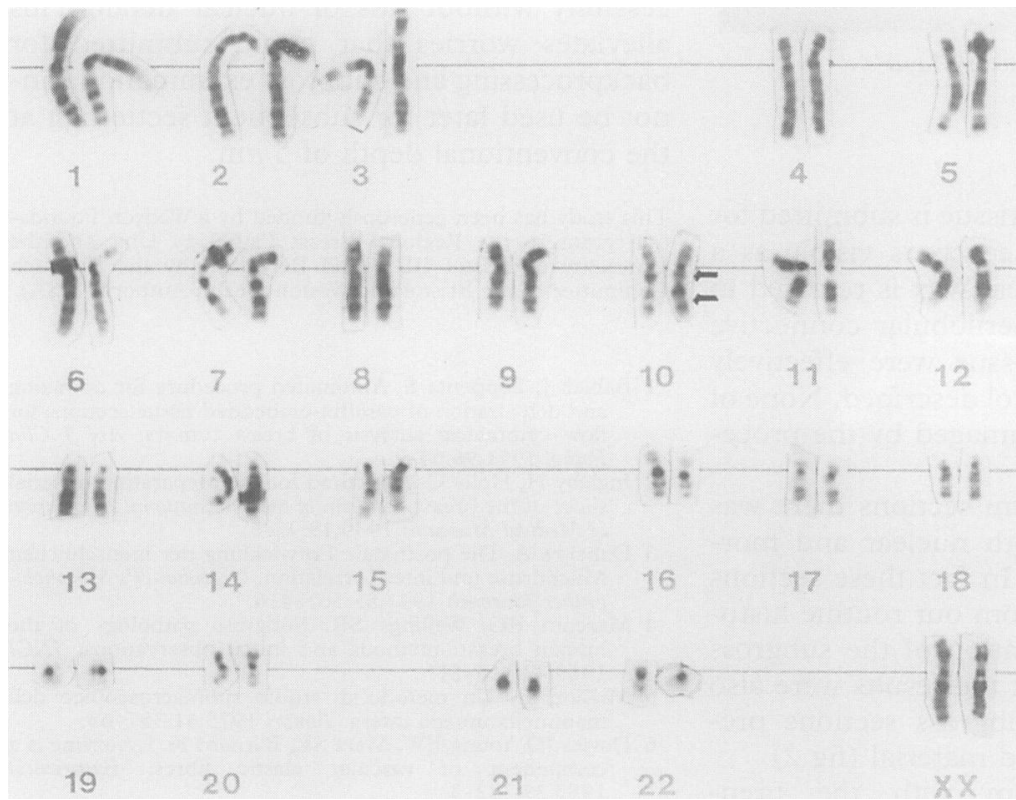

G-banded karyotype from the peripheral blood of a patient with T-chronic lymphocytic leukaemia showing the inversion of chromosome 10, inv(10) (q11q24). three to seven days; others incubate the slides for 24 hours at $65^{\circ} \mathrm{C}$ in a dessicator. Either of these strategies does not avoid the cytogenetic result being delayed and therefore less useful for diagnostic and clinical purposes. We modified a simple technique to obtain good G-banding on the same day as the slides are prepared, using a microwave oven.

\section{Methods}

The slides were placed immediately, or at most one to two hours after being made, in a microwave oven which was programmed at the highest intensity for five minutes. Afterwards the slides were stained for three minutes with Wright's stain according to the method of Yunis $^{2}$ diluted 1 in 3 in Sörensen buffer.

We also tested specimens as we normally do in our laboratory (waiting at least three or four days), and stained the preparations before putting them into the microwave oven the same day as they were prepared.

We checked the slides made from more than 100 bone marrow samples, and from more than 25 peripheral blood samples from patients with chronic lymphoproliferative disorders and also from amniotic fluid. In most of these samples we obtained equally good quality bands in the older preparations as in the preparations placed in the microwave. But the slides stained directly did not show bands or the bands were of poor resolution.

The figure is an example of the technical quality accomplished. It shows a karyotype from the peripheral blood of a patient with chronic lymphocytic leukaemia with a satisfactory banding resolution, the tiny inversion on the long arm of chromosome 10 being easily detected.

\section{Conclusion}

This simple modification can produce satisfactory chromosome banding on fresh samples rapidly making clinically useful results quicker to obtain.

1 Boon ME, Kok LP. In: The art of microscopic visualization. In: The microwave cookbook of pathology. Leyden: Coulom Press, 1989.

2 Yunis JJ. New chromosome technique in the study of human neoplasia. Hum Pathol 1981;12:540-9. 